\title{
Polarization coupling in single-mode single-polarization optical fibers
}

\author{
Chao-Xiang Shi and Rong-Qing Hui \\ Optical Communication Laboratory, P.O. Box 27, Radio Engineering Department, Beijing University of Posts and Telecommunications, \\ Beijing, China
}

Received March 18, 1988; accepted September 13, 1988

Polarization coupling in single-mode, single-polarization optical fibers is investigated, and a set of coupled-mode equations for single-mode, single-polarization fibers including guided and leaky modes is derived.

In 1983, Snyder and Ruhl predicted that fibers composed of highly birefringent material have a singlepolarization property because of leaky-mode effects..$^{1,2}$ Varnham et al. then published their elaborate experimental results that affirmed that the difference in bend loss between the two polarized modes is the predominant mechanism of single polarization in a practical bow-tie fiber. ${ }^{3,4} \mathrm{~A}$ recent paper indicates that the tilting of the anisotropic axis in single-mode, singlepolarization (SMSP) fibers can amplify their singlepolarization effect. ${ }^{5}$ In this Letter we present a theoretical analysis on the mode-coupling effect in SMSP optical fibers.

Marcuse $^{6}$ analyzed mode coupling in planar anisotropic waveguides, but such structures do not exhibit the tunneling leaky modes ${ }^{1,2}$ for nonplanar geometries. This Letter addresses coupling between guided and tunneling leaky modes. When an optical fiber undergoes external perturbations, polarization coupling is induced, and there is continuous exchange of energy between the guided and leaky modes. The guided mode couples part of its energy to the leaky mode, which in turn can couple back a small part of its energy to the guided mode. The leaky mode also leaks some energy. to the cladding; therefore, the energy carried by the guided mode dissipates through polarization mode coupling.

The wave equation in an anisotropic material is

$$
\nabla^{2} \mathbf{E}+\omega^{2} \mu \vec{\epsilon} \mathbf{E}=\nabla(\nabla \cdot \mathbf{E}) .
$$

First, we consider a reference optical fiber without external perturbations: both the core and the cladding are made of an anisotropic material with the principal refractive-index axes aligned and taken to be $n_{x}, n_{y}$, and $n_{z}$, so that dielectric tensors take the form

$$
\vec{\epsilon}=\epsilon_{0}\left[\begin{array}{lll}
n_{x}{ }^{2} & 0 & 0 \\
0 & n_{y}{ }^{2} & 0 \\
0 & 0 & n_{z}{ }^{2}
\end{array}\right]=\left[\begin{array}{ll}
\ddot{\epsilon}_{t} & 0 \\
0 & \epsilon_{z}
\end{array}\right],
$$

where

$$
\ddot{\epsilon}_{t}=\left[\begin{array}{cc}
\epsilon_{0} n_{x}^{2} & 0 \\
0 & \epsilon_{0} n_{y}^{2}
\end{array}\right], \quad \epsilon_{z}=\epsilon_{0} n_{z}^{2}
$$

and $\epsilon_{0}$ denotes the dielectric permittivity in vacuum.

We can express the electrical field in terms of its transverse and longitudinal components $\mathbf{E}=\mathbf{E}_{t}+\mathbf{E}_{z}$, substitute it into Eq. (1), and use the condition

$$
\nabla^{2}=\nabla_{t}^{2}+\frac{\partial^{2}}{\partial z^{2}}, \quad \nabla \cdot(\vec{\epsilon} \cdot \mathbf{E})=0
$$

to derive the following wave equation for the transverse components:

$$
\begin{aligned}
\nabla_{t}^{2} \mathbf{E}_{t}+\omega^{2} \mu \vec{\epsilon}_{t} \cdot \mathbf{E}_{t} & +\frac{\partial^{2}}{\partial z^{2}} \mathbf{E}_{t} \\
& =\nabla_{t} \nabla_{t} \mathbf{E}_{t}-\frac{1}{\epsilon_{0} n_{z}{ }^{2}} \nabla_{t} \nabla_{t} \cdot\left(\vec{\epsilon}_{t} \cdot \mathbf{E}_{t}\right) .
\end{aligned}
$$

In Cartesian coordinates, $\mathbf{E}_{t}=\left(e_{x} \mathbf{x}+\right.$ $\left.e_{y} y\right) \exp (-j \beta z)$, therefore we have the following separated equations:

$$
\begin{aligned}
& {\left[\nabla_{t}^{2}+\left(k_{0} n_{x}\right)^{2}-\beta^{2}\right] e_{x}=\frac{\partial}{\partial x}\left(P_{x} e_{x}+P_{y} e_{y}\right),} \\
& {\left[\nabla_{t}^{2}+\left(k_{0} n_{y}\right)^{2}-\beta^{2}\right] e_{y}=\frac{\partial}{\partial y}\left(P_{x} e_{x}+P_{y} e_{y}\right),}
\end{aligned}
$$

where $k_{0}=\omega \sqrt{\mu \epsilon_{0}}$ and

$$
\begin{aligned}
& P_{x} e_{x}=\left[1-\left(\frac{n_{x}}{n_{z}}\right)^{2}\right] \frac{\partial e_{x}}{\partial x}-\left(\frac{n_{x}}{n_{z}}\right)^{2} e_{x} \frac{\partial}{\partial x}\left(\ln n_{x}{ }^{2}\right), \\
& P_{y} e_{y}=\left[1-\left(\frac{n_{y}}{n_{z}}\right)^{2}\right] \frac{\partial e_{y}}{\partial y}-\left(\frac{n_{y}}{n_{z}}\right)^{2} e_{y} \frac{\partial}{\partial y}\left(\ln n_{y}{ }^{2}\right) .
\end{aligned}
$$

We also have an equation for the longitudinal component:

$$
\left[\nabla_{t}^{2}+\left(k_{0} n_{z}\right)^{2}-\beta^{2}\right] e_{z}=j \beta\left(P_{x} e_{x}+P_{y} e_{y}\right) .
$$


Snyder has solved the above equations with the Green function method?:

$$
\begin{aligned}
& \mathbf{E}_{t 1}=\mathbf{e}_{t 1} \exp \left(-j \beta_{y} z-\alpha z\right)= {\left[e_{y 1} \mathbf{y}+e_{x 1}(\Delta) \mathbf{x}\right] } \\
& \times \exp \left[-\left(j \beta_{y}-\alpha\right) z\right], \quad(3 \mathrm{a}) \\
& \mathbf{E}_{t 2}=\mathbf{e}_{t 2} \exp \left(-j \beta_{x} z\right)=\left[e_{x 2} \mathbf{x}+e_{y 2}(\Delta) \mathbf{y}\right] \exp \left(-j \beta_{x} z\right) .
\end{aligned}
$$

Here $\mathbf{E}_{t 1}$ is the $y$-polarization mode (assumed to be leaky), $\alpha$ is the leaky loss coefficient of the $y$-polarization mode, $\mathbf{E}_{t 2}$ is the $x$-polarization mode (assumed to be guided), and $e_{x 1}(\Delta)$ and $e_{y 2}(\Delta)$ are the small components along the $x$ and $y$ directions of the $y$ - and $x$ polarization modes, respectively. Analytical expressions for $e_{y 1}, e_{x 1}(\Delta), e_{y 2}(\Delta), e_{x 2}$, and $\alpha$ have been derived.?

We now consider the impact of external perturbations on SMSP fibers (core deformation, bending, microbending, etc.). Any perturbation can be made equivalent to a fluctuation of the dielectric tensors. In this Letter only transverse fluctuation of the index profile is taken into account because of its dominant effect on mode coupling in practical stress-applying optical fibers. ${ }^{8}$ The dielectric tensor of a perturbed fiber is

$$
\overrightarrow{\tilde{\epsilon}}=\epsilon_{0}\left[\begin{array}{ccc}
\tilde{n}_{x x}{ }^{2} & \tilde{n}_{x y}{ }^{2} & 0 \\
\tilde{n}_{y x}{ }^{2} & \tilde{n}_{y y}{ }^{2} & 0 \\
0 & 0 & 0
\end{array}\right]=\left[\begin{array}{cc}
\ddot{\tilde{\epsilon}}_{t} & 0 \\
0 & \epsilon_{z}
\end{array}\right],
$$

From Eqs. (1) and (2) we have the wave equation for the transverse component of a perturbed fiber:

$$
\begin{aligned}
\nabla_{t}^{2} \mathbf{E}_{t}+\omega^{2} \mu \ddot{\tilde{\epsilon}}_{t} \cdot \mathbf{E}_{t} & +\frac{\partial^{2}}{\partial z^{2}} \mathbf{E}_{t} \\
& =\nabla_{t} \nabla_{t} \cdot \mathbf{E}_{t}-\frac{1}{\epsilon_{0} \tilde{n}_{z}{ }^{2}} \nabla_{t} \nabla_{t} \cdot\left(\ddot{\tilde{\epsilon}}_{t} \cdot \mathbf{E}_{t}\right) .
\end{aligned}
$$

$\mathbf{E}_{t}$ can be expressed as the superposition of the ideal modes $\mathbf{E}_{t m}$ of the reference SMSP fiber,

$$
\begin{aligned}
\mathbf{E}_{t}=\sum_{m} C_{m}(z) \mathbf{E}_{t m}=\sum_{m} C_{m}(z) \mathbf{e}_{t m} \exp \left(\lambda_{m} z\right) & \\
m & =1,2,
\end{aligned}
$$

where $\mathbf{E}_{t 1}$ and $\mathbf{E}_{t 2}$ represent the reference SMSP fiber's $y$-polarization mode and $x$-polarization mode, respectively. The analytical expression for $E$ can be deduced from Eqs. (3) with $\lambda_{1}=-j \beta_{y}-\alpha$ and $\lambda_{2}=$ $-j \beta_{x} . \quad C_{1}(z)$ and $C_{2}(z)$ describe the mode amplitude and phase. To derive the coupled-mode equation that only includes the variables $C_{1}(z)$ and $C_{2}(z)$, we must utilize the orthogonality of ideal modes of the reference SMSP fiber. The orthogonality conditions for two polarization modes of the reference SMSP fiber can be developed as follows.

We begin by substituting Eqs. (3) into Eq. (2),

$$
\begin{array}{r}
\nabla_{t}^{2} \mathbf{e}_{t m}{ }^{*}+\omega^{2} \mu \ddot{\epsilon}_{t}^{+} \cdot \mathbf{e}_{t m}{ }^{*}+\lambda_{m}{ }^{2 *} \mathbf{e}_{t m}{ }^{*} \\
=\nabla_{t} \nabla_{t} \cdot \mathbf{e}_{t m}{ }^{*}-\frac{1}{\epsilon_{0} n_{z}{ }^{2}} \nabla_{t} \nabla_{t} \cdot\left(\vec{\epsilon}_{t}^{+} \cdot \mathbf{e}_{t m}{ }^{*}\right), \\
\nabla_{t}^{2} \mathbf{e}_{t n}+\omega^{2} \mu \vec{\epsilon}_{t} \cdot \mathbf{e}_{t n}+\lambda_{n}{ }^{2} \mathbf{e}_{t n} \\
=\nabla_{t} \nabla_{t} \cdot \mathbf{e}_{t n}-\frac{1}{\epsilon_{0} n_{z}{ }^{2}} \nabla_{t} \nabla_{t} \cdot\left(\vec{\epsilon}_{t} \cdot \mathbf{e}_{t n}\right) .
\end{array}
$$

Here $\mathbf{e}_{t m} *$ is the complex conjugate of the vector $\mathbf{e}_{t m}$ and $\vec{\epsilon}^{+}$is the complex conjugate of the transpose of a tensor $\vec{\epsilon}_{t}$. We form the dot product of Eq. (6) with $\mathbf{e}_{t n}$ and of Eq. (7) with $\mathbf{e}_{t m}{ }^{*}$, then integrate over the infinite cross section and subtract the resulting two equations. Because the reference fiber is lossless, i.e., $\vec{\epsilon}_{t}{ }^{+}=$ $\vec{\epsilon}_{t}$, we use

$$
\nabla_{t}^{2} \mathbf{A}=\nabla_{t} \nabla_{t} \cdot \mathbf{A}-\nabla_{t} \times \nabla_{t} \times \mathbf{A}
$$

to yield

$$
\begin{aligned}
& \left(\lambda_{m}{ }^{2 *}-\lambda_{n}^{2}\right) \iint_{A_{\infty}} \mathbf{e}_{t n} \cdot \mathbf{e}_{t m}{ }^{*} \mathrm{~d} s \\
& =\iint_{A_{\infty}}\left(\mathbf{e}_{t n} \cdot \nabla_{t} \times \nabla_{t} \times \mathbf{e}_{t m}{ }^{*}-\mathbf{e}_{t m}{ }^{*} \cdot \nabla_{t} \times \nabla_{t} \times \mathbf{e}_{t n}\right) \mathrm{d} s \\
& =\frac{1}{\epsilon_{0} n_{z}^{2}} \iint_{A_{\infty}}\left[\mathbf{e}_{t n} \cdot \nabla_{t} \nabla_{t} \cdot\left(\vec{\epsilon}_{t}^{+} \cdot \mathbf{e}_{t m}{ }^{*}\right)\right. \\
& \left.\quad-\mathbf{e}_{t m}{ }^{*} \cdot \nabla_{t} \nabla_{t} \cdot\left(\ddot{\epsilon}_{t} \cdot \mathbf{e}_{t n}\right)\right] \mathrm{d} s .
\end{aligned}
$$

To prove the orthogonality conditions, the following equations are used:

$$
\begin{aligned}
& \nabla_{t} \cdot\left[\mathbf{e}_{t m}{ }^{*} \times\left(\nabla_{t} \times \mathbf{e}_{t n}\right)\right]-\nabla_{t} \cdot\left[\mathbf{e}_{t n} \times\left(\nabla_{t} \times \mathbf{e}_{t m}{ }^{*}\right)\right] \\
& =\mathbf{e}_{t n} \cdot \nabla_{t} \times \nabla_{t} \times \mathbf{e}_{t m}{ }^{*}-\mathbf{e}_{t m}{ }^{*} \cdot \nabla_{t} \times \nabla_{t} \times \mathbf{e}_{t n}, \\
& \nabla_{t} \cdot\left[\mathbf{e}_{t n} \nabla_{t} \cdot\left(\vec{\epsilon}_{t}^{+} \cdot \mathbf{e}_{t m}^{*}\right)-\nabla_{t} \cdot\left[\mathbf{e}_{t m}{ }^{*} \nabla_{t} \cdot\left(\vec{\epsilon}_{t} \cdot \mathbf{e}_{t n}\right)\right]\right. \\
& =\mathbf{e}_{t n} \cdot \nabla_{t} \nabla_{t} \cdot\left(\ddot{\epsilon}_{t}^{+} \cdot \mathbf{e}_{t m}{ }^{*}\right)-\mathbf{e}_{t m}{ }^{*} \cdot \nabla_{t} \nabla_{t} \cdot\left(\vec{\epsilon}_{t} \cdot \mathbf{e}_{t n}\right) \text {. }
\end{aligned}
$$

With the above equations, the surface integral becomes a line integral,

$$
\begin{aligned}
& \left(\lambda_{m}^{2 *}-\lambda_{n}{ }^{2}\right) \iint_{A_{\infty}} \mathbf{e}_{t n} \cdot \mathbf{e}_{t m} \mathrm{~d} s \\
& =\int_{l \infty}\left[\mathbf{e}_{t m}{ }^{*} \times\left(\nabla_{t} \times \mathbf{e}_{t n}\right)-\mathbf{e}_{t n} \times\left(\nabla_{t} \times \mathbf{e}_{t m}{ }^{*}\right)\right] \cdot \mathrm{d} \mathbf{l}-\frac{1}{\epsilon_{0} n_{z}^{2}} \\
& \quad \times \int_{l \infty}\left[\mathbf{e}_{t n} \nabla_{t} \cdot\left(\vec{\epsilon}_{t}{ }^{+} \cdot \mathbf{e}_{t m}{ }^{*}\right)-\mathbf{e}_{t m}{ }^{*} \nabla \cdot\left(\vec{\epsilon}_{t} \cdot \mathbf{e}_{t n}\right)\right] \cdot \mathrm{d} \mathbf{l} .
\end{aligned}
$$

We know that the $y$-polarization mode is leaky, with its $x$ component oscillatory in the cladding. However, the $x$-polarization mode is guided, and thus it decays exponentially in the cladding. We conclude that the line integral along a closed path that approaches infinity will vanish. This leads to the orthogonality condition

$$
\iint_{A_{\infty}} \mathbf{e}_{t m} * \cdot \mathbf{e}_{t n} \mathrm{~d} s=0, \quad m \neq n
$$


Substituting Eq. (5) into Eq. (4), and neglecting the influence of the second-order variables $\partial^{2} C_{m} / \partial z^{2}$, we obtain two sets of equations: the wave equation satisfied by ideal modes of the reference fiber (i.e., a wave equation of the SMSP fiber without external perturbations) and the coupled-mode equation of the two polarization modes including $C_{1}(z)$ and $C_{2}(z)$ :

$$
\begin{aligned}
& \sum_{m}\left(2 \lambda_{m} \frac{\mathrm{d} C_{m}}{\mathrm{~d} z} \mathbf{e}_{t m}+\omega^{2} \mu \Delta \vec{\epsilon}_{t} \cdot \mathbf{e}_{t m} C_{m}+\right.\left.\Delta \vec{P} \cdot \mathbf{e}_{t m} C_{m}\right) \\
& \times \exp \left(\lambda_{m} z\right)=0,
\end{aligned}
$$

where

$$
\Delta \vec{\epsilon}_{t}=\ddot{\tilde{\epsilon}}_{t}-\vec{\epsilon}_{t}
$$

and

$$
\begin{aligned}
\Delta \ddot{P} \cdot \mathbf{e}_{t m}=\left(\frac{1}{\epsilon_{0} \tilde{n}_{z}^{2}} \nabla_{t} \nabla_{t} \cdot \ddot{\tilde{\epsilon}}_{t}-\frac{1}{\epsilon_{0} n_{z}^{2}} \nabla_{t} \nabla_{t} \vec{\epsilon}_{t}\right) \cdot \mathbf{e}_{t m} & \\
& m=1,2 .
\end{aligned}
$$

Utilizing the orthogonality condition of Eq. (8), we derive the coupled-mode equations

$$
\begin{aligned}
& \frac{\mathrm{d} C_{1}}{\mathrm{~d} z}=k_{11} C_{1}+k_{12} C_{2} \exp \left(j \beta_{y}-j \beta_{x}+\alpha\right) z, \\
& \frac{\mathrm{d} C_{2}}{\mathrm{~d} z}=k_{21} C_{1} \exp \left(j \beta_{x}-j \beta_{y}-\alpha\right) z+k_{22} C_{2},
\end{aligned}
$$

where

$$
\begin{gathered}
k_{m n}=\left(I_{m n}^{[1]}+I_{m n}^{[2]}\right) / 2 \lambda_{m} Q_{m}, \\
I_{m n}^{[1]}=\omega^{2} \mu \iint_{A_{\infty}} \mathbf{e}_{t m} \cdot\left(\Delta \ddot{\epsilon}_{t} \cdot \mathbf{e}_{t n}^{*}\right) \mathrm{d} s \\
I_{m n}^{[2]}=\iint_{A_{\infty}} \mathbf{e}_{t m} \cdot\left(\Delta \mathrm{P} \cdot \mathbf{e}_{t n}^{*}\right) \mathrm{d} s
\end{gathered}
$$

$$
Q_{m}=\iint_{A_{\infty}} \mathbf{e}_{t m} \cdot \mathbf{e}_{t m}^{*} \mathrm{~d} s, \quad n, m=1,2 .
$$

In the above derivation, the cladding of the anisotropic fiber is infinitely extended so that the integral over the squared magnitude of the leaky mode diverges. However, in practical fibers, the cladding is finitely extended and the outer-cladding boundary forces the field of the leaky mode to decay rapidly. Thus, we need only to substitute the infinite integral section $A_{\infty}$ by the finite cross section of the practical fiber when calculating the above integrals.

We have derived the coupled-mode equations of SMSP fibers. Because the propagation constant of the leaky mode is complex and the $x$ component of the leaky mode is oscillatory in the cladding, the coefficients of the coupled differential equations become complex. However, the coupling coefficients in ordinary single-mode fibers and polarization-maintaining fibers (two polarizations) are purely real or imaginary. This indicates that the guided mode also has a loss through polarization coupling in SMSP fibers.

The authors are grateful to A. W. Snyder for his advice and encouragement.

\section{References}

1. A. W. Snyder and F. Ruhl, Electron. Lett. 19, 185 (1983).

2. A. W. Snyder and F. Ruhl, J. Opt. Soc. Am. 73, 1165 (1983).

3. M. D. Varnham, D. N. Payne, R. D. Birch, and E. J. Tarbox, Electron. Lett. 19, 246 (1983).

4. M. D. Varnham, D. N. Payne, R. D. Birch, and E. J. Tarbox, Electron. Lett. 19, 679 (1983).

5. A. W. Snyder and A. Ankiewicz, J. Opt. Soc. Am. A 3, 856 (1986).

6. D. Marcuse, Bell Syst. Tech. J. 54, 985 (1975).

7. A. W. Snyder and F. Ruhl, IEEE J. Lightwave Technol. LT-2, 284 (1984).

8. K. Okamoto, Y. Sasaki, and N. Shibata, IEEE J. Quantum Electron. QE-18, 1890 (1982). 